\title{
Reentrant nematic and smectic A phases : the dielectric approach
}

\author{
L. Benguigui \\ Solid State Institute, Technion-Israel Institute of Technology, Haifa, Israel \\ and F. Hardouin \\ Centre de Recherches Paul-Pascal, Université Bordeaux-I, 33405 Talence Cedex, France
}

(Reçu le 26 novembre 1980, révisé le 19 janvier 1981, accepté le 19 janvier 1981)

\begin{abstract}
Résumé. - Ceci représente la première étude des propriétés diélectriques du phénomène doublement rentrant $\mathrm{N}-\mathrm{S}_{\mathrm{A}}-\mathrm{N}-\mathrm{S}_{\mathrm{A}}$. Ces mesures sont en bon accord avec une précédente analyse structurale par rayons $\mathrm{X}$. Les deux phases smectiques A révèlent des comportements.différents, aussi bien en ce qui concerne leurs constantes diélectriques que leur temps de relaxation parallèle. De plus, la phase nématique rentrante apparaît liée à la coexistence de deux sortes d'organisation smectique à courte distance, l'une caractéristique de la phase smectique $\mathrm{A}$ basse température, et l'autre de la phase smectique $\mathbf{A}$ haute température.
\end{abstract}

\begin{abstract}
We report the first dielectric investigations of the double reentrant phenomenon N-S $-\mathrm{N}_{\mathrm{A}}-\mathrm{S}_{\mathrm{A}}$. These measurements are in good agreement with an earlier X-Ray analysis. Both smectic A phases present very different behaviours in their dielectric constants as well as in their parallel relaxation times. In addition, the reentrant nematic can be seen as the simultaneous presence of two types of smectic short range order, one characterizing the low temperature $S_{A}$ and the other the high temperature smectic $A$.
\end{abstract}

1. Introduction. - In recent years, liquid crystal reentrant phenomena have been evidenced in many rod-like polar systems. First, the appearance of a reentrant nematic phase was revealed in mixtures of two cyano compounds [1], then in cyano derivatives under high pressure (like 8OCB or CBOOA) [2] and, at last, even in pure cyano materials at atmospheric pressure [3, 4]. In this latter case, the 4-n-octyloxybenzoyloxy-4'-cyano stilbene ( $\mathrm{T} 8$ » for short) was the first material investigated, and it has the very particular property to exhibit in fact two reentrant phases : a nematic one and a smectic $A$ one [3]. The different transition temperatures and the chemical formula are :

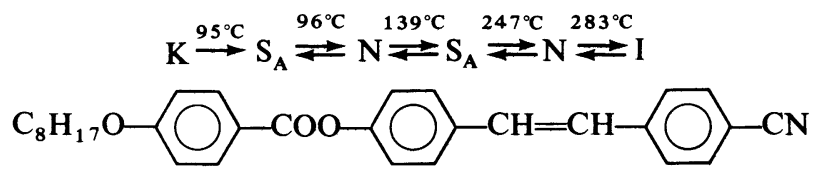

X-ray study [5] of this compound has shown that the two smectic phases have different short range structures. In the high temperature smectic A phase
(H.T. $\mathrm{S}_{\mathrm{A}}$ ), we have the well known partially bilayer structure, common to numerous cyano compounds [6] with the strong polar $\mathrm{CN}$ group : the layer thickness $d$ is larger than the molecular length $l$ in its most extended form $(d / l \sim 1.3)$. This structure is generally explained by a $S_{A}$ packing with molecular cores overlapping. However, in the low temperature smectic A phase (L.T. $\mathrm{S}_{\mathrm{A}}$ ) the layer thickness is nearly equal to the molecular length $(d / l \simeq 0.99)$. Furthermore, in this phase unusual X-ray diffuse scatterings appear which are consistent with a two dimensional short range superlattice periodicity depicted in reference [5]. A tentative microscopic explanation is also presented [5] with groups of parallel molecules and others with antiparallel molecules.

In this paper, we present dielectric measurements on " $\mathrm{T} 8$ " : dielectric constants which are very sensitive to the short range dipole ordering, especially in the smectic phases [7], and dielectric relaxations which can give informations on the dynamical properties.

2. Experimental results. - The quasi static dielectric constants $\varepsilon_{\|}$and $\varepsilon_{\perp}$ has been measured at $15 \mathrm{kHz}$ by means of the automatic bridge LC 1688 of General 
Radio. The parallel dielectric relaxation with the measuring electric field parallel to the long molecular axis is measured by means of a $Q$ meter of Boonton.

We performed measurements between $180^{\circ} \mathrm{C}$ and $80^{\circ} \mathrm{C}$ i.e. in the two smectic $\mathrm{A}$ phases and in the reentrant nematic phase. Because of the high temperature of the upper nematic phase, we could not measure the dielectric properties in this phase. However, following the data given in reference [8] and our unpublished measurements on CBOOA it is likely that the dielectric properties in the upper nematic phase are similar to those of other liquid crystals without reentrant phenomena.

The sample was orientated in the reentrant nematic phase using a magnetic field of $13 \mathrm{kG}$, afterward we performed the measurements.

On figure 1 , we show the temperature dependence of $\varepsilon_{\|}$and $\varepsilon_{\perp}$. There are slight variation in $\varepsilon_{\perp}$, with a maximum at about $112^{\circ} \mathrm{C}$. For $\varepsilon_{\|}$, we note the maximum in the reentrant nematic phase itself around $120^{\circ} \mathrm{C}$. This important change in the nematic phase contrasts with the minor changes which appear at the two $\mathrm{N} \mathrm{S}_{\mathrm{A}}$ transitions : at $140{ }^{\circ} \mathrm{C}$ for $\varepsilon_{\|}$and at $96^{\circ} \mathrm{C}$ for $\varepsilon_{\perp}$. This clearly indicates that there is no important variation in the short range order at the $\mathrm{N} \mathrm{S}_{\mathrm{A}}$ transitions in good agreement with the fact that these transitions are quasi second order [3].

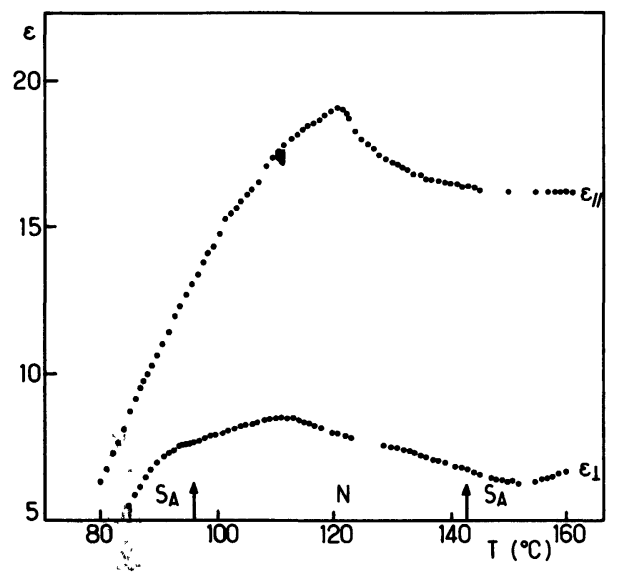

Fig. 1. - Quasi static permittivity components $\varepsilon_{\|}$and $\varepsilon_{\perp}(15 \mathrm{kHz})$ of $\mathrm{T} 8$ at different temperatures.

The dynamical measurements will help us in the understanding of the particular behaviour of $\varepsilon_{\|}$in the reentrant nematic phase.

On figure 2 we draw the variation of the tangent of the dielectric loss angle as function of the frequency, at various temperatures, and on figure 3 we show the variation of the logarithm of the relaxation frequency $f_{\mathrm{R}}$ as a function of $1 / T$. Note that $f_{\mathrm{R}}$ is not the maximum of the tangent of the dielectric loss angle, but is the maximum of $\varepsilon_{2}$, the imaginary part of the complex permittivity $\varepsilon=\varepsilon_{1}-j \varepsilon_{2}$. The interesting results is that we can distinguish on the figures $2-3$ the presence of two regimes : one is characteristic of

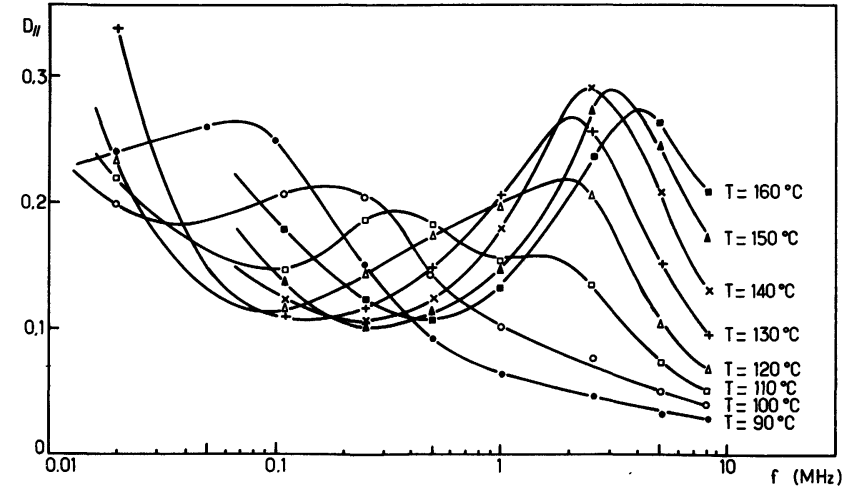

Fig. 2. - Tangent of the dielectric loss angle $(\mathbf{E} / / \mathbf{n})$ at different frequencies.

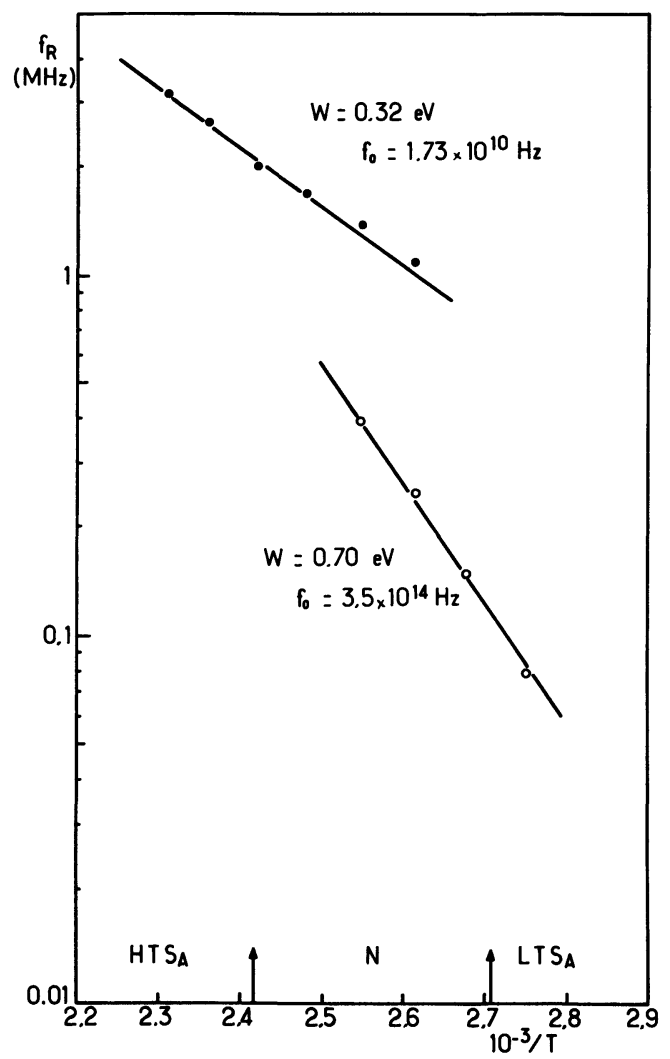

Fig. 3. - Relaxation frequency versus inverse of the temperature $f_{\mathrm{R}}=f_{0} \exp (-W / k T)$.

the L.T. $S_{A}$ phase (with higher values of the relaxation time $\tau=1 / 2 \pi f_{\mathrm{R}}$ ) and the second of the H.T. $\mathrm{S}_{\mathrm{A}}$ phase (with lower values of $\tau$ ). In the nematic phase we have either one of the two regimes or coexistence of the two regimes around $110-120^{\circ} \mathrm{C}$. It is just the temperature range in which the maxima in $\varepsilon_{\|}$and $\varepsilon_{\perp}$ appear. We remark the conitinuity of $f_{\mathrm{R}}$ at the two transition temperatures. This is in disagreement with the results of B. R. Ratna et al. [8] who observe a discontinuity in $f_{\mathrm{R}}$ at the $\mathrm{S}_{\mathrm{A}}$-reentrant nematic. However, they performed measurements on samples with only a reentrant nematic phase. If we express the relaxation time $\tau=1 / 2 \pi f_{\mathrm{R}}$ as $\tau=\tau_{0} \exp (W / R T)$ we 
get in the $S_{\mathrm{A}}$ phase $\tau_{0} \simeq 5 \times 10^{-16} \mathrm{~s}$ and $W=0.70 \mathrm{eV}$ and in the H.T. $\mathrm{S}_{\mathrm{A}} \tau_{0} \simeq 10^{-11} \mathrm{~s}$ and $W=0.32 \mathrm{eV}$.

3. Interpretation. - Our results give a beautiful confirmation of the structural results of $F$. Hardouin and A. M. Levelut [5] : the two smectic A phases have two different structures and this is reflected in the dielectric measurements. We observe two very different relaxation times and $\varepsilon_{\|}$behaves also very differently in the two smectic phases : in the H.T. $S_{A}$ phase $\varepsilon_{\|}$is almost constant, but it decreases strongly with temperature in the L.T. $S_{A}$ phase.

3.1 THE SMECTIC PHASES. - In fact $\varepsilon_{\|}$decreases slowly also in the H.T. $\mathrm{S}_{\mathrm{A}}$. The decrease of $\varepsilon_{\|}$with $T$ in the smectic phases is in agreement with the general belief [7] that in the smectic phase there are antiparallel correlations in the direction parallel to the long molecular axis. In our case, the partially bilayer structure is also explained by the antiparallel configuration of the molecules. However, the interesting point is that $\left|\mathrm{d} \varepsilon_{\|} / \mathrm{d} T\right|$ is larger in the L.T. $S_{\mathrm{A}}$ phase than in the H.T. $S_{A}$ phase. This may be merely explained by an increase in the smectic order parameter, nevertheless a more detailed analysis of the dipolar interaction between the molecules in the two structures is necessary in order to understand this difference in $\left|\mathrm{d} \varepsilon_{\|} / \mathrm{d} T\right|$. But we can already say that the strong decrease of $\varepsilon_{\|}$ in the L.T. $S_{A}$ does not give support of a microscopic picture which assumes that there exist regions where the molecules are parallel [5]. From these dielectric results a short range antiparallel configuration with strong antiferroelectric correlations is more likely.

We saw above that $W$ is larger and $\tau_{0}$ smaller in the L.T. $S_{A}$ phase than in the H.T. $S_{A}$ phase. It is not easy to explain these differences since at the present stage there is no satisfactory theory of the relaxation in liquid crystals. However, it is known that $\tau$ is related to two important quantities [9] : a) the height of the nematic barrier; $b) D_{\perp}$, one of the diffusion coefficients. Our results could be understood from the H.T. $S_{A}$ to the L.T. $S_{A}$ by a decrease of the diffusion coefficient and/or by an increase of the nematic barrier. Intuitively, one can say that the partially bilayer structure of the H.T. $S_{A}$ phase is more open than the monolayer structure of the L.T. $S_{A}$ and this may be the explanation why it is easier for a molecule to flip around a short axis in the H.T. $S_{A}$ phase than in the L. T. $S_{A}$ phase. It is also very likely that this property is related to the antiferroelectric correlations : the stronger they are, the larger the relaxation time is, as observed (see Figs. 1 and 3).

3.2 The ReEntrant nematic Phase. - The presence of the maximum of $\varepsilon_{\|}$in this phase is easily explained using the fact that the antiferroelectric correlations are larger in a smectic phase than in a nematic phase [10]. Thus, starting from the H.T. $\mathrm{S}_{A}$ phase and decreasing the temperature, $\varepsilon_{\|}$increases after crossing the H.T. $S_{A}-N$ transition temperature because the smectic short range order will be smaller and smaller. However, approaching the second $\mathrm{N}-\mathrm{S}_{\mathrm{A}}$ transition, the smectic short range order begins to increase again resulting in a decrease of $\varepsilon_{\|}$with $T$. The relaxation time measurements show the existence of two types of smectic short range order, one characterizing the L.T. $S_{A}$ phase and the other the H.T. $S_{A}$ phase. This is in complete agreement with the Hardouin and Levelut X-ray results [5] : in the reentrant nematic phase, on the high temperature side, there are cybotactic groups of partially bilayer $S_{A}$ and on the low temperature side, cybotactic groups of monolayer $\mathrm{S}_{\mathrm{A}}$. And around $120^{\circ} \mathrm{C}$, our results show the coexistence of the two types of cybotactic groups which is quite confirmed by recent X-ray experiments on an homologous compound of the T 8 exhibiting the same reentrant sequence $\mathrm{N}-\mathrm{S}_{\mathrm{A}}-\mathrm{N}-\mathrm{S}_{\mathrm{A}}$ [11]. Let us note that the existence of two different modes to explain the behaviour of such polar systems has been developed in J. Prost's theory [12].

4. Conclusion. - The dielectric measurements we have performed on T 8 are in very good agreement with the earlier structural X-ray measurements [5]. In particular, the reentrant nematic phase can be seen as a consequence of the matching of two different $S_{A}$ fluctuations. Concerning the two smectic A phases, very different behaviours are observed in their dielectric constants as well as in their parallel relaxation time. We claim that the antiparallel correlations are stronger in the low temperature $S_{A}$ phase than in the high temperature $S_{A}$ one and the relaxation appears smaller in the low temperature $S_{A}$ with a different activation energy.

\section{References}

[1] Cladis, P. E., Phys. Rev. Lett. 35 (1975) 48.

[2] Bogardus, P. E., Bogardus, R. K., Daniels, W. B., Taylor, G. N., Phys. Rev. Lett. 39 (1977) 720.

[3] Hardouin, F., Sigaud, G., Achard, M. F., Gasparoux, H., Phys. Lett. 71A (1979) 347; Solid State Commun. 30 (1979) 265.

[4] Madhusudana, N. V., Sadashiva, B. K., Moodithaya, K. P. L., Current Sci. 48 (1979) 613.

[5] Hardouin, F., Levelut, A. M., J. Physique 41 (1980) 41.

[6] Leadbetter, A. J., Frost, J. C., Gaughan, J. P., Gray, G. W. Mosley, A., J. Physique 40 (1979) 375.

[7] Benguigui, L., J. Physique 40 (1979) 705
[8] Ratna, B. R., Shashidhar, R., Rao, K. V., presented at the Int. Liq. Crystals Conf. Bangalore, 1979.

[9] Luckhurst, G. R., Yeates, R. Y., Chem. Phys. Lett. 38 (1976) 551

[10] De Jeu, W. H., Goossens, W. J. A., BordewiJk, P., J. Chem Phys. 61 (1974) 1985.

[11] Hardouin, F., Levelut, A. M., Sigaud, G., J. Physique 42 (1981) 71.

[12] Prost, J., Proceedings of the Conference on Liquid Crystals of one- and two-dimensional order : Garmisch-Partenkirchen (Springer Verlag, Berlin, Heidelberg, New York) 1980, p. 125. 